\title{
Effects of green manures during fallow on moisture and nutrients of soil and winter wheat yield on the Loss Plateau of China
}

\author{
Naiwen Xue, Zhenping Yang*, Zhiqiang Gao*, Chunlai Zhang, Jianfu Xue, Xiaoli Liu, Min Sun, Tianqing Du \\ Crop Ecology and Dry Cultivation Physiology Key Laboratory of Shanxi Province in College of Agronomy, Shanxi Agricultural University, \\ Taigu 030801, Shanxi, People's Republic of China
}

\section{A B S T R A C T}

The application of catch crops as a green manure can enhance soil fertility owing to improving soil nutrients. We conducted one year field experiment to evaluate the effect of catch crops [Rapeseed (Brassica napus L.) under different sowing rates and Soy bean (G/ycine max L.)] with biological organic fertilizer $1,500 \mathrm{~kg} / \mathrm{ha}$ on wheat yield and soil nutrients. The green manures were sown on $3^{\text {th }} \mathrm{July} 2015$ during summer fallow. At the beginning, there were five treatments as follows: R1 (Rapeseed and sowing rate was $7.5 \mathrm{~kg} / \mathrm{ha}$ ); R2 (Rapeseed and sowing rate was $15 \mathrm{~kg} / \mathrm{ha}$ ); R3 (Rapeseed and sowing rate was $22.5 \mathrm{~kg} / \mathrm{ha}$ ); S (Soy bean and sowing rate was $105 \mathrm{~kg} / \mathrm{ha}$ ); C (Control was bare field). Every green manure treatment was split into two treatments on $27^{\text {th }}$ September 2015. One treatment we turned the above ground biomass of green manure into the soil. And another treatment we harvested the above ground biomass of green manure. The above ground biomass turned into soil treatments were G1, G3, G5 and G7. The above ground biomass harvested treatments were G2, G4, G6 and G8. The treatment $C$ was still the bare field. Soil samples were taken twice to measure soil moisture and nutrients at two stages. One stage was in autumn before winter wheat sowing and another stage was in next year summer after wheat harvest. The significantly highest 1,000-grain weight and grain yield belonged to the treatment $\mathrm{C}$, which were $10.69 \%-36.87 \%$ and $16.86 \%-72.5 \%$ higher than that of the green manures treatments. After wheat harvest, the $0-20 \mathrm{~cm}$ soil available $\mathrm{N}$ and total $\mathrm{N}$ of $\mathrm{G} 7$ were $51.40 \%-20.45 \%$ and $95.12 \%-125.35 \%$ significantly better than that of other treatments. The significantly highest soil total P of $0-20 \mathrm{~cm}$ belonged to $\mathrm{G} 3$ after wheat harvest, which was $25 \%-45.83 \%$ better than other treatments. Before wheat sowing, the treatment $\mathrm{C}$ kept the significantly lowest soil available $\mathrm{K}$ of five layers. The $0-20 \mathrm{~cm}$ and $20-40 \mathrm{~cm}$ soil organic matter of the treatment $\mathrm{S}$ was $40.28 \%-71.12 \%$ and $53.92 \%-122.67 \%$ significantly higher than other treatments before wheat sowing. Therefore, growing rapeseed and soy bean during summer fallow in this region significantly reduced 1,000-grain weight and grain yield of subsequent winter wheat. But the incorporation of green manures improved the soil nutrients to some extent Abstract: The application of catch crops as a green manure can enhance soil fertility owing to improving soil nutrients. We conducted one year field experiment to evaluate the effect of catch crops [Rapeseed (Brassica napus L.) under different sowing rates and Soy bean (Glycine max L.)] with biological organic fertilizer 1,500 kg/ha on wheat yield and soil nutrients. The green manures were sown on $3^{\text {th }}$ July 2015 during summer fallow. At the beginning, there were five treatments as follows: R1 (Rapeseed and sowing rate was $7.5 \mathrm{~kg} / \mathrm{ha}$ ); R2 (Rapeseed and sowing rate was $15 \mathrm{~kg} / \mathrm{ha}$ ); R3 (Rapeseed and sowing rate was $22.5 \mathrm{~kg} / \mathrm{ha}$ ); S (Soy bean and sowing rate was $105 \mathrm{~kg} / \mathrm{ha}$ ); C (Control was bare field). Every green manure treatment was split into two treatments on $27^{\text {th }}$ September 2015. One treatment we turned the above ground biomass of green manure into the soil. And another treatment we harvested the above ground biomass of green manure. The above ground biomass turned into soil treatments were $\mathrm{G} 1$, G3, G5 and G7. The above ground biomass harvested treatments were G2, G4, G6 and G8. The treatment C was still the bare field. Soil samples were taken twice to measure soil moisture and nutrients at two stages. One stage was in autumn before winter wheat sowing and another stage was in next year summer after wheat harvest. The significantly highest 1,000-grain weight and grain yield belonged to the treatment $\mathrm{C}$, which were $10.69 \%-36.87 \%$ and $16.86 \%-72.5 \%$ higher than that of the green manures treatments. After wheat harvest, the $0-20 \mathrm{~cm}$ soil available $\mathrm{N}$ and total N of G7 were $51.40 \%-20.45 \%$ and $95.12 \%-125.35 \%$ significantly better than that of other treatments. The significantly highest soil total P of $0-20 \mathrm{~cm}$ belonged to $\mathrm{G} 3$ after wheat harvest, which was $25 \%-45.83 \%$ better than other treatments. Before wheat sowing, the treatment $\mathrm{C}$ kept the significantly lowest soil available $\mathrm{K}$ of five layers. The $0-20 \mathrm{~cm}$ and $20-40 \mathrm{~cm}$ soil organic matter of the treatment $\mathrm{S}$ was $40.28 \%-71.12 \%$ and $53.92 \%-122.67 \%$ significantly higher than other treatments before wheat sowing. Therefore, growing rapeseed and soy bean during summer fallow in this region significantly reduced 1,000-grain weight and grain yield of subsequent winter wheat. But the incorporation of green manures improved the soil nutrients to some extent.

Keywords: Green manures; Summer fallow; Soil moisture; Soil nutrients; Wheat yield

\footnotetext{
*Corresponding author:

Zhenping Yang. College of Agronomy, Shanxi Agricultural University, Taigu 030801, Shanxi, People's Republic of China.

E-mail: yangzp.2@163.com/Zhiqiang Gao. College of Agronomy, Shanxi Agricultural University, Taigu 030801, Shanxi, People's Republic of China. E-mail: gaozhiqiang1964@126.com
}

Received: 01 March 2017; $\quad$ Revised: 03 January 2018;

Accepted: 09 January 2018;

Published Online: 12 January 2018 


\section{INTRODUCTION}

The Loess Plateau of China is a typical rain-fed region of intensive agricultural development and crop production, which is gradually facing the serious problem of soil nutrient shortage (Li et al., 2009). More than $60 \%$ of the annual precipitation falls on this area when wheat fields are fallow (usually from July to September) because of the continental monsoon climate (Jin et al. 2007). Tang (2004) stated that severe wind and water erosion, unsuitable cultivation systems, and a serious lack of organic fertilizer lead to soil quality degradation, fragile physical structure, and low soil organic matter content in this area. Therefore, how to relieve the wind and water erosion and increase soil nutrients during the fallow period is critical for the subsequent wheat yield.

The selection of a catch crop utilized as a green manure mainly depends on the primary aims achieved (Hwang et al., 2015). Legumes can fix atmospheric N (Hargrove, 1986), reduce weeds (Krishnan et al., 1998; Norsworthy et al., 2005), enhance soil physical and biological properties, and improve soil carbon and N cycling (Tejada et al., 2008; Lee et al., 2010; Piotrowska and Wilczewski, 2012). In addition, non-legumes are mainly applied to prevent soil erosion, accumulate nutrients, decrease nutrient leaching losses, and enhance the soil organic carbon storage (Vos and van der Putten, 2001; Poeplau et al., 2015). Soybean is a widely used leguminous green manure in China. Rapeseed has a high aboveground biomass and high $\mathrm{N}$, $\mathrm{P}$ and $\mathrm{K}$ content (Hu et al., 2015). Thus, soybean and rapeseed are selected as green manures in our research.
Furthermore, most previous studies of green manures effect on subsequent crop yield and soil fertility focus on humid regions and seldom study concentrates on dry land area. Therefore, we conducted an experiment on the Loss Plateau of China. And we hypothesized that (1) growing green manures during summer fallow would increase grain yield of subsequent winter wheat better than the bare field treatment, (2) growing green manures would improve more soil relative water content before wheat sowing and after wheat harvest than the bare field treatment, (3) the soil nutrients would be enhanced by green manures more before wheat sowing and after wheat harvest than the bare field treatment.

\section{MATERIAL AND METHODS}

\section{Site description and experimental design}

The study site was located at Qiujialing Village (latitude $35^{\circ} 09^{\prime} \mathrm{N}$ and longitude $\left.110^{\circ} 59^{\prime} \mathrm{E}\right)$, Wenxi County, Shanxi Province, China. The cropping system consisted of winter wheat (always from October to next June) and a summer fallow (always from middle of June to the end of September). The study site was on the southeast of Loess Plateau and had a continental monsoon climate. From 2005 to 2016, the annual precipitation of the study site was $450.53 \mathrm{~mm}$, and averagely $58.8 \%$ fell during the summer fallow (Fig. 1). From 2005 to 2016, the annual average air temperature was $13.4^{\circ} \mathrm{C}$. The maximum mean monthly temperature is $26.4^{\circ} \mathrm{C}$ (in July), and the minimum mean monthly temperature is $-0.8^{\circ} \mathrm{C}$ (in January). From 2015 to 2016, the annual precipitation of the study site

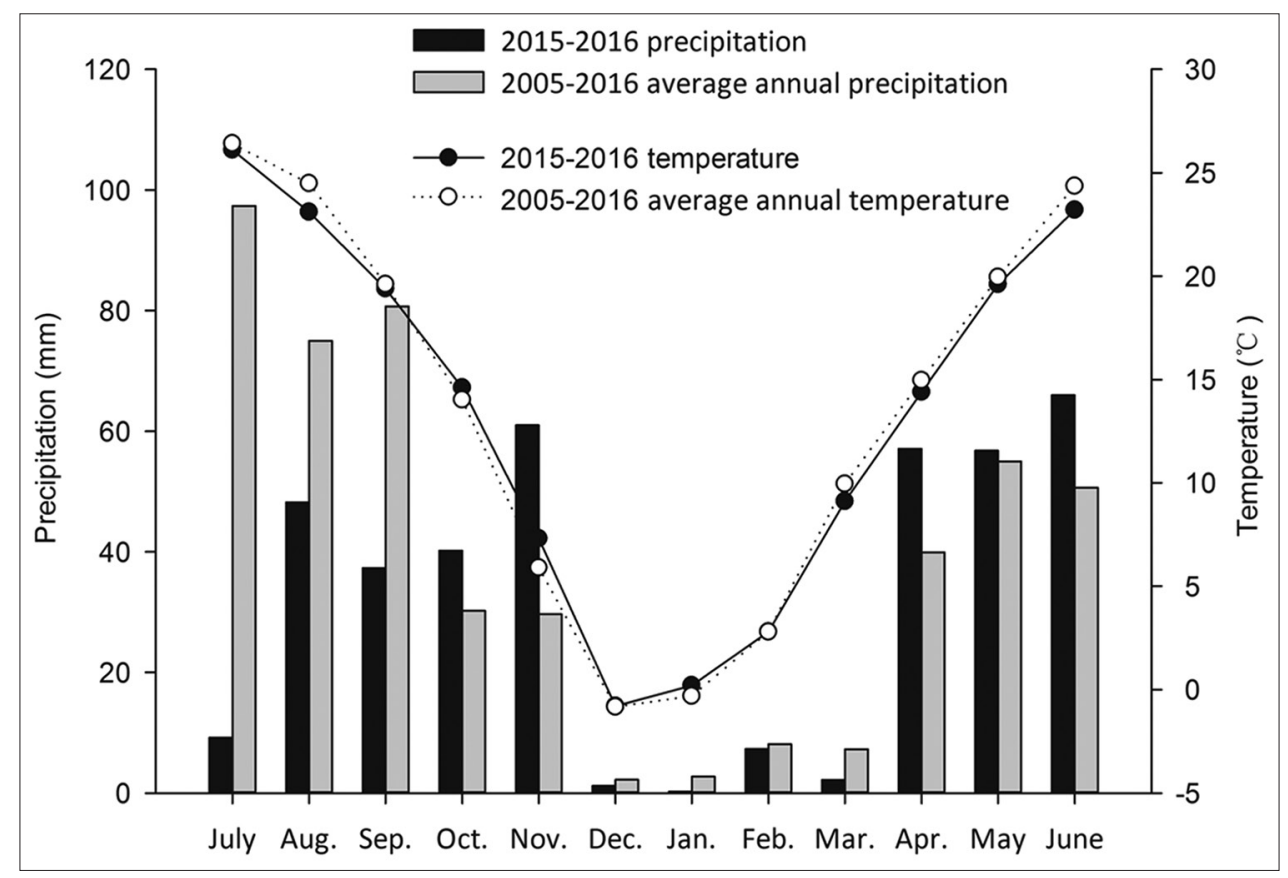

Fig 1. Weather conditions at experimental site Qiujialing Village, Wenxi County, Shanxi Province, China, from 2015 to 2016. 
was $386.8 \mathrm{~mm}$, and $94.7 \mathrm{~mm}$ fell during the summer fallow (Fig. 1). The experimental field had no irrigation equipment. The soil at the study site was sandy loam. The chemical properties of the soil before green manures were sown were given in Table 1.

The experiment was conducted from $1^{\text {th }}$ July 2015 to $10^{\text {th }}$ June 2016. The trial applied a randomized block design. The plot size was $1.5 \mathrm{~m} \times 3 \mathrm{~m}=34.5 \mathrm{~m}^{2}$ and was replicated three times. The previous wheat was harvest on $15^{\text {th }}$ June 2015. And the stubbles of wheat were left $20-30 \mathrm{~cm}$ high. Biological organic fertilizer was applied on July $1^{\text {st }} 2015$, at a rate of $1,500 \mathrm{~kg} / \mathrm{ha}$. We utilized the fertilizer brand 'Wofeng', with $\geq 2 \times 10^{8} / \mathrm{g}$ effective live bacteria, $\geq 25 \%$ organic matter and $\geq 65 \% \mathrm{~N}+\mathrm{P}_{2} \mathrm{O}_{5}+\mathrm{K}_{2} \mathrm{O}$. Wheat stubbles and organic fertilizer was applied to the soil with a deep turning machine, choosing a depth of $25-30 \mathrm{~cm}$. After the first rain of wheat harvest, the green manures were sown on $3^{\text {th }}$ July 2015 . The treatments were as follows: R1 (Rapeseed and sowing rate was $7.5 \mathrm{~kg} / \mathrm{ha}$ ); R2 (Rapeseed and sowing rate was $15 \mathrm{~kg} / \mathrm{ha}$ ); R3 (Rapeseed and sowing rate was $22.5 \mathrm{~kg} / \mathrm{ha}$ ); S (Soy bean and sowing rate was $105 \mathrm{~kg} / \mathrm{ha}$ ). The rapeseed cultivar was Huayouza 6 and the soybean cultivar was Jinda 78 . The control was bare field (C). The treatment $C$ was carried out deep tillage $(35-40 \mathrm{~cm})$ by large tractor after wheat harvest. Every green manure treatment was split into two treatments on $27^{\text {th }}$ September. One treatment we turned the above ground biomass of green manure into the soil. And another treatment we harvested the above ground biomass of green manure. The above ground biomass turned into soil treatments were G1, G3, G5 and G7. The above ground biomass harvested treatments were G2, G4, G6 and G8. Every treatment size was $17.25 \mathrm{~m}^{2}$ and replicated three times. The control was still bare field (C). Basic urea, $\mathrm{P}_{2} \mathrm{O}_{5}$ and $\mathrm{K}_{2} \mathrm{O}$ were spread manually at the rate of $150 \mathrm{~kg} / \mathrm{ha}$ on $27^{\text {th }}$ September 2015. The fertilizers and green manure biomass were rotated into the soil by a large tractor on $27^{\text {th }}$ September 2015 . And the rotary tillage depth was $15-20 \mathrm{~cm}$. The winter wheat (Jinmai 47) was sown by a machine as ridges with film and furrows. The seeds were planted in the furrows. Line space in the furrows was $20 \mathrm{~cm}$ and line space between the ridges was $40 \mathrm{~cm}$. The highest point of the covering film was $10 \mathrm{~cm}$. The sowing density was $97.5 \mathrm{~kg} / \mathrm{ha}$. The experiment design was shown in Table 2.

\section{Plant and soil analysis}

At maturity, $16 \mathrm{~m}^{2}$ of wheat were harvested manually from each plot to calculate spike number per hectare, grain number per spike, 1,000-grain weight and grain yield. The harvest index (HI) was calculated as the fraction of grain dry matter divided by the total aboveground biomass on a per hectare basis.

Soils were sampled at a depth from $0 \mathrm{~cm}$ to $100 \mathrm{~cm}$, with $20 \mathrm{~cm}$ intervals. Three soil cores were taken from each plot, using an auger with an inner diameter of $4 \mathrm{~cm}$. All samples from one plot were placed in a labeled plastic bag, mixed, and sealed to obtain one composite sample per plot. Prior to the analysis of total $\mathrm{N}$, total $\mathrm{P}$, available $\mathrm{N}, \mathrm{P}$, and $\mathrm{K}$, the samples were air-dried. Soil samples for the determination of soil water were collected in aluminum soil boxes; all boxes were weighed immediately to obtain fresh weights. The samples were dried at $105^{\circ} \mathrm{C}$ to constant weight and

Table 1: Basic soil nutrients before green manures were sow

\begin{tabular}{|c|c|c|c|c|c|c|}
\hline $\begin{array}{l}\text { Soil } \\
\text { depth }(\mathrm{cm})\end{array}$ & $\begin{array}{c}\text { Available N } \\
\text { (mg/kg) }\end{array}$ & Total N ( $\mathbf{g} / \mathbf{k g})$ & Available P (mg/kg) & Total P $(\mathbf{g} / \mathbf{k g})$ & Available K (mg/kg) & Organic matter (\%) \\
\hline $0-20 \mathrm{~cm}$ & $36.47 \pm 2.47$ & $0.91 \pm 0.09$ & $1.42 \pm 0.26$ & $1.00 \pm 0.03$ & $206.14 \pm 10.80$ & $4.09 \pm 0.31$ \\
\hline $20-40 \mathrm{~cm}$ & $27.20 \pm 1.24$ & $0.78 \pm 0.05$ & $1.57 \pm 0.40$ & $0.87 \pm 0.05$ & $138.20 \pm 13.06$ & $4.02 \pm 0.79$ \\
\hline $40-60 \mathrm{~cm}$ & $19.78 \pm 1.24$ & $0.71 \pm 0.04$ & $0.47 \pm 0.18$ & $0.78 \pm 0.03$ & $98.57 \pm 5.66$ & $3.03 \pm 0.50$ \\
\hline $60-80 \mathrm{~cm}$ & $16.07 \pm 0.62$ & $0.75 \pm 0.05$ & $0.34 \pm 0.01$ & $0.78 \pm 0.03$ & $91.77 \pm 3.00$ & $2.50 \pm 0.18$ \\
\hline $80-100 \mathrm{~cm}$ & $20.39 \pm 1.85$ & $0.65 \pm 0.03$ & $0.34 \pm 0.06$ & $0.77 \pm 0.02$ & $97.43 \pm 3.00$ & $3.65 \pm 0.40$ \\
\hline
\end{tabular}

The values are mean values $\pm S D(n=3)$.

Table 2: The experiment design of green manures growth period and wheat growth period

\begin{tabular}{|c|c|c|c|}
\hline \multicolumn{2}{|c|}{ Green manures growth period } & \multicolumn{2}{|c|}{ Wheat growth period } \\
\hline Treatment & Crops & Treatment & Harvested or composted green manures before wheat sowing \\
\hline \multirow[t]{2}{*}{ R1 } & Rapeseed $(7.5 \mathrm{~kg} / \mathrm{ha})$ & G1 & Composted \\
\hline & & G2 & Harvested \\
\hline \multirow[t]{2}{*}{$\mathrm{R} 2$} & Rapeseed (15 kg/ha) & G3 & Composted \\
\hline & & G4 & Harvested \\
\hline \multirow[t]{2}{*}{ R3 } & Rapeseed $(22.5 \mathrm{~kg} / \mathrm{ha})$ & G5 & Composted \\
\hline & & G6 & Harvested \\
\hline \multirow[t]{2}{*}{ S } & Soy bean (105 kg/ha) & G7 & Composted \\
\hline & & G8 & Harvested \\
\hline C & Bare field & C & Bare field \\
\hline
\end{tabular}


weighed again to obtain gravimetric water content. Total $\mathrm{N}$ was measured using the sulfuric acid digestion and Kjeldahl distillation (Lu, 2000). Available $\mathrm{N}$ was measured using the alkali-diffusion method. Total $\mathrm{P}$ was measured using the sulfuric acid-Perchloric acid digestion method, while available $\mathrm{P}$ was extracted with $0.5 \mathrm{M} \mathrm{NaHCO}_{3}$. Available $\mathrm{K}$ was extracted with $1.0 \mathrm{M} \mathrm{NH}_{4} \mathrm{OA}$. Soil organic matter was measured by wet digestion using potassium dichromate along with a mixture of $\mathrm{H}_{2} \mathrm{SO}_{4}$ and $85 \% \mathrm{H}_{3} \mathrm{PO}_{4}$ (Snyder and Trofymow, 1984).

\section{Statistical analysis}

Statistical analysis was conducted using the SAS 8.1 software package. The data were analyzed by one-way ANOVA with treatment means tested by the least significant range (LSR) method at a significance level of $\mathrm{P}<0.05$. The figure was drawn with SigmaPlot 12.0 (Systat Software Inc., San Jose, CA, USA).

\section{RESULTS}

\section{Grain yield and three components of grain yield of winter wheat}

There were no significant differences of spike number per hectare and grain number per spike among treatments (Table 3). But the treatment $\mathrm{C}$ held a higher trend of spike number per hectare and grain number per spike than other treatments. The significantly highest 1,000-grain weight belonged to the treatment $\mathrm{C}$ which was $10.69 \%-36.87 \%$ higher than 1,000-grain weight in the green manures treatments. And the treatment $\mathrm{C}$ also led to the significantly highest grain yield, which was 16.86\%-72.5\% higher than the grain yield in the grain manures treatment. The treatments G3, G4, G5 and G6 obtained a higher trend of 1,000-grain weight and grain yield than that of $G 7$ and G8. Highest biomass values were found in the treatments G8 and C, however the lowest biomass belonged to G1. The treatment G5 resulted in the significantly highest HI and G8 obtained the lowest HI.

\section{Soil relative water content}

The treatment $C$ led to a higher trend of soil relative water content of $0-100 \mathrm{~cm}$ than the green manures treatments before wheat sowing (Table 4). However, R3 held a lower trend of soil water content of $0-100 \mathrm{~cm}$ compared with other treatments before wheat sowing. With the sowing rate of rapeseed increasing, the soil water content of 0-100 $\mathrm{cm}$ had a decreasing trend. In $0-20 \mathrm{~cm}$ soil layer, the water content of the treatment $C$ was $8.9 \%-47.06 \%$ higher than that of green manures treatments. At $40-60 \mathrm{~cm}$ and 60-80 cm soil layers, there was only significant difference between the treatment $C$ and R3. In $80-100 \mathrm{~cm}$ soil layer, the water content of the treatment $\mathrm{C}$ was significantly higher than that of R1 and R3. After wheat harvest, the treatment $\mathrm{C}$ held a higher trend of soil water content at $0-20 \mathrm{~cm}$ and was $4.33 \%-51.47 \%$ higher than other treatment.

\section{Soil nutrients \\ Soil available $N$}

Table 5 shows that the treatment $\mathrm{S}$ obtained a higher trend of available $\mathrm{N}$ from 0 to $60 \mathrm{~cm}$ before wheat sowing compared with other treatments, which indicated that planting soy bean could increase available $\mathrm{N}$ from top to medium depth of soil. And the $0-20 \mathrm{~cm}$ available $\mathrm{N}$ of the treatment $\mathrm{S}$ was $5.88 \%-20.01 \%$ higher than that of other treatments. After wheat harvest, the significantly highest available $\mathrm{N}$ of $0-20 \mathrm{~cm}$ was found in $\mathrm{G} 7$, which was $51.40 \%-20.45 \%$ better than other treatments. In addition, G7 also had the highest available $\mathrm{N}$ at $60-80 \mathrm{~cm}$.

\section{Soil Total N}

Before wheat sowing, the treatment $S$ held a higher trend of soil total $\mathrm{N}$ from 20 to $100 \mathrm{~cm}$ than other treatments (Table 6), which indicated that planting soy bean could increase soil total $\mathrm{N}$ at these soil layers. After wheat harvest, at $0-20 \mathrm{~cm}$, the significantly highest soil total $\mathrm{N}$ was observed in G7, which was $95.12 \%-125.35 \%$ higher than other treatments.

Table 3: Three components of yield, grain yield, biomass and harvest index

\begin{tabular}{lcccccc} 
Treatment & $\begin{array}{c}\text { Spike number per } \\
\text { hectare } \\
\left(\times 10^{6} \text { number/ha) }\right.\end{array}$ & $\begin{array}{c}\text { Grain number } \\
\text { per spike } \\
\text { (number/spike) }\end{array}$ & $\begin{array}{c}1000 \text {-grain } \\
\text { weight }(\mathbf{g})\end{array}$ & $\begin{array}{c}\text { Grain yield } \\
(\mathbf{m g} / \text { ha) }\end{array}$ & $\begin{array}{c}\text { Aboveground } \\
\text { biomass (mg/ha) }\end{array}$ & $\begin{array}{c}\text { Harvest } \\
\text { index }(\%)\end{array}$ \\
G1 & $3.82 \pm 0.24^{\mathrm{a}}$ & $30.27 \pm 1.12^{\mathrm{a}}$ & $40.76 \pm 0.20^{\mathrm{c}}$ & $4.69 \pm 0.25^{\mathrm{d}}$ & $13.69 \pm 0.44^{\mathrm{e}}$ & $0.34 \pm 0.01^{\mathrm{c}}$ \\
G2 & $3.52 \pm 0.75^{\mathrm{a}}$ & $32.42 \pm 2.76^{\mathrm{a}}$ & $50.40 \pm 0.04^{\mathrm{ab}}$ & $4.58 \pm 0.87^{\mathrm{d}}$ & $16.16 \pm 0.02^{\text {cd }}$ & $0.28 \pm 0.00^{\mathrm{d}}$ \\
G3 & $3.70 \pm 0.51^{\mathrm{a}}$ & $33.97 \pm 2.47^{\mathrm{a}}$ & $46.82 \pm 0.38^{\mathrm{abc}}$ & $6.03 \pm 0.63^{\mathrm{bc}}$ & $21.41 \pm 0.19^{\mathrm{b}}$ & $0.28 \pm 0.00^{\mathrm{d}}$ \\
G4 & $4.03 \pm 0.73^{\mathrm{a}}$ & $32.30 \pm 1.19^{\mathrm{a}}$ & $47.45 \pm 0.46^{\mathrm{abc}}$ & $6.18 \pm 0.86^{\mathrm{b}}$ & $17.77 \pm 0.47^{\mathrm{c}}$ & $0.35 \pm 0.01^{\mathrm{c}}$ \\
G5 & $4.17 \pm 0.43^{\mathrm{a}}$ & $26.86 \pm 3.15^{\mathrm{a}}$ & $46.74 \pm 0.57^{\mathrm{abc}}$ & $6.76 \pm 0.50^{\mathrm{b}}$ & $16.49 \pm 0.75^{\text {cd }}$ & $0.41 \pm 0.02^{\mathrm{a}}$ \\
G6 & $4.07 \pm 0.84^{\mathrm{a}}$ & $31.10 \pm 2.13^{\mathrm{a}}$ & $49.66 \pm 0.26^{\mathrm{abc}}$ & $6.31 \pm 0.97^{\mathrm{b}}$ & $16.73 \pm 0.14^{\text {cd }}$ & $0.38 \pm 0.00^{\mathrm{b}}$ \\
G7 & $3.84 \pm 0.34^{\mathrm{a}}$ & $31.05 \pm 3.15^{\mathrm{a}}$ & $42.68 \pm 0.50^{\mathrm{bc}}$ & $5.11 \pm 0.38^{\mathrm{cd}}$ & $15.50 \pm 0.35^{\mathrm{d}}$ & $0.33 \pm 0.01^{\mathrm{c}}$ \\
G8 & $3.35 \pm 0.12^{\mathrm{a}}$ & $33.48 \pm 3.01^{\mathrm{a}}$ & $42.65 \pm 0.12^{\mathrm{bc}}$ & $4.81 \pm 0.35^{\mathrm{d}}$ & $30.12 \pm 1.01^{\mathrm{a}}$ & $0.16 \pm 0.01^{\mathrm{e}}$ \\
C & $4.21 \pm 0.21^{\mathrm{a}}$ & $34.06 \pm 3.56^{\mathrm{a}}$ & $55.79 \pm 0.18^{\mathrm{a}}$ & $7.90 \pm 0.28^{\mathrm{a}}$ & $28.62 \pm 0.07^{\mathrm{a}}$ & $0.28 \pm 0.00^{\mathrm{d}}$ \\
\hline
\end{tabular}

The values are mean values $\pm S D(n=3)$, Different lowercased letters in one column indicate a significant difference with $P<0.05$. 
Table 4: Soil relative water content at two stages (\%)

\begin{tabular}{|c|c|c|c|c|c|}
\hline \multicolumn{6}{|c|}{ Soil relative water content before wheat sowing (\%) } \\
\hline Treatment & $0-20 \mathrm{~cm}$ & $20-40 \mathrm{~cm}$ & $40-60 \mathrm{~cm}$ & $60-80 \mathrm{~cm}$ & $80-100 \mathrm{~cm}$ \\
\hline R1 & $7.21 \pm 0.37^{\mathrm{bc}}$ & $7.09 \pm 0.30^{\mathrm{a}}$ & $7.36 \pm 0.35^{\mathrm{ab}}$ & $7.28 \pm 0.46^{\mathrm{ab}}$ & $7.13 \pm 0.38^{\mathrm{bc}}$ \\
\hline $\mathrm{R} 2$ & $9.18 \pm 0.35^{\mathrm{ab}}$ & $8.59 \pm 0.18^{\mathrm{a}}$ & $8.19 \pm 0.50^{\mathrm{ab}}$ & $7.74 \pm 0.10^{\mathrm{ab}}$ & $7.63 \pm 0.74^{\mathrm{ab}}$ \\
\hline R3 & $6.80 \pm 0.37^{c}$ & $6.98 \pm 0.54^{\mathrm{a}}$ & $6.54 \pm 0.29^{b}$ & $6.53 \pm 0.18^{\mathrm{b}}$ & $6.50 \pm 0.14^{c}$ \\
\hline S & $7.94 \pm 0.97^{\mathrm{abc}}$ & $7.09 \pm 0.42^{\mathrm{a}}$ & $7.92 \pm 0.40^{\mathrm{ab}}$ & $7.34 \pm 0.09^{\mathrm{ab}}$ & $7.81 \pm 0.38^{\mathrm{ab}}$ \\
\hline $\mathrm{C}$ & $10.00 \pm 0.14^{\mathrm{a}}$ & $9.33 \pm 0.27^{\mathrm{a}}$ & $9.17 \pm 0.54^{a}$ & $8.66 \pm 0.35^{a}$ & $8.35 \pm 0.88^{a}$ \\
\hline \multicolumn{6}{|c|}{ Soil relative water content after wheat harvest (\%) } \\
\hline Treatment & $0-20 \mathrm{~cm}$ & $20-40 \mathrm{~cm}$ & $40-60 \mathrm{~cm}$ & $60-80 \mathrm{~cm}$ & $80-100 \mathrm{~cm}$ \\
\hline G1 & $13.33 \pm 0.36^{\mathrm{ab}}$ & $12.12 \pm 1.24^{\mathrm{a}}$ & $6.52 \pm 0.78^{\mathrm{bc}}$ & $6.35 \pm 0.78^{\mathrm{bcd}}$ & $6.33 \pm 0.78^{\mathrm{bc}}$ \\
\hline G2 & $14.78 \pm 0.57^{\mathrm{a}}$ & $12.92 \pm 0.77^{\mathrm{a}}$ & $9.08 \pm 0.23^{a}$ & $8.21 \pm 0.89^{a}$ & $7.10 \pm 0.89^{\mathrm{ab}}$ \\
\hline G3 & $14.23 \pm 0.32^{\mathrm{a}}$ & $12.48 \pm 1.86^{\mathrm{a}}$ & $6.86 \pm 0.25^{\mathrm{bc}}$ & $6.95 \pm 0.03^{\mathrm{abc}}$ & $6.37 \pm 0.03^{\mathrm{bc}}$ \\
\hline G4 & $10.18 \pm 1.23^{b}$ & $7.16 \pm 1.34^{b}$ & $5.82 \pm 0.80^{c}$ & $5.19 \pm 0.17^{d}$ & $5.89 \pm 0.17 c$ \\
\hline G5 & $12.66 \pm 1.31^{\mathrm{ab}}$ & $7.97 \pm 1.64^{\mathrm{b}}$ & $6.68 \pm 0.40^{\mathrm{bc}}$ & $6.42 \pm 0.26^{\mathrm{bcd}}$ & $6.30 \pm 0.26^{\mathrm{bc}}$ \\
\hline G6 & $12.42 \pm 1.88^{\mathrm{ab}}$ & $7.06 \pm 1.12^{b}$ & $6.41 \pm 0.23^{b c}$ & $5.73 \pm 0.70^{\text {cd }}$ & $6.12 \pm 0.70^{\circ}$ \\
\hline G7 & $14.56 \pm 0.97^{a}$ & $11.51 \pm 1.01^{\mathrm{a}}$ & $9.95 \pm 0.66^{a}$ & $8.48 \pm 0.13^{a}$ & $7.41 \pm 0.13^{\mathrm{a}}$ \\
\hline G8 & $10.92 \pm 1.04^{b}$ & $7.42 \pm 0.70^{\mathrm{b}}$ & $6.85 \pm 0.17^{\mathrm{bc}}$ & $4.92 \pm 0.60^{d}$ & $4.73 \pm 0.60^{d}$ \\
\hline C & $15.42 \pm 0.55^{a}$ & $12.51 \pm 2.46^{\mathrm{a}}$ & $8.33 \pm 0.56^{\mathrm{ab}}$ & $7.63 \pm 0.50^{\mathrm{ab}}$ & $7.79 \pm 0.50^{\mathrm{a}}$ \\
\hline
\end{tabular}

The values are mean values $\pm \mathrm{SD}(\mathrm{n}=3)$. Different lowercased letters in one column indicate a significant difference with $P<0.05$.

Table 5: Soil available $\mathrm{N}(\mathrm{mg} / \mathrm{kg})$ at two stages

\begin{tabular}{|c|c|c|c|c|c|}
\hline \multicolumn{6}{|c|}{ Soil available $\mathrm{N}(\mathrm{mg} / \mathrm{kg})$ before wheat sowing } \\
\hline Treatment & $0-20 \mathrm{~cm}$ & $20-40 \mathrm{~cm}$ & $40-60 \mathrm{~cm}$ & $60-80 \mathrm{~cm}$ & $80-100 \mathrm{~cm}$ \\
\hline $\mathrm{R} 1$ & $42.03 \pm 1.63^{\mathrm{ab}}$ & $37.08 \pm 1.07^{\mathrm{a}}$ & $29.66 \pm 2.83^{\mathrm{ab}}$ & $25.34 \pm 4.05^{a}$ & $37.70 \pm 1.63^{a}$ \\
\hline $\mathrm{R} 2$ & $37.08 \pm 1.07^{b}$ & $33.99 \pm 2.23^{\mathrm{ab}}$ & $23.49 \pm 1.63^{\mathrm{bc}}$ & $19.16 \pm 4.83^{\mathrm{a}}$ & $38.94 \pm 4.28^{a}$ \\
\hline R3 & $40.79 \pm 1.85 a^{b}$ & $38.94 \pm 3.86^{\mathrm{a}}$ & $29.67 \pm 0.00^{\mathrm{ab}}$ & $42.03 \pm 2.55^{\mathrm{a}}$ & $40.66 \pm 3.79^{a}$ \\
\hline S & $44.50 \pm 2.14^{\mathrm{a}}$ & $42.03 \pm 3.76^{a}$ & $32.76 \pm 2.47^{a}$ & $28.43 \pm 3.52^{\mathrm{a}}$ & $47.59 \pm 4.18^{\mathrm{a}}$ \\
\hline C & $42.02 \pm 0.62^{\mathrm{ab}}$ & $27.81 \pm 1.86^{b}$ & $22.87 \pm 1.23^{c}$ & $22.25 \pm 3.71^{\mathrm{a}}$ & $53.15 \pm 2.56^{\mathrm{a}}$ \\
\hline \multicolumn{6}{|c|}{ Soil available $\mathrm{N}(\mathrm{mg} / \mathrm{kg})$ after wheat harvest } \\
\hline Treatment & $0-20 \mathrm{~cm}$ & $20-40 \mathrm{~cm}$ & $40-60 \mathrm{~cm}$ & $60-80 \mathrm{~cm}$ & $80-100 \mathrm{~cm}$ \\
\hline G1 & $33.37 \pm 0.00^{c}$ & $37.08 \pm 1.86^{a}$ & $25.96 \pm 3.71^{\mathrm{ab}}$ & $20.40 \pm 1.86^{c}$ & $19.47 \pm 0.93^{\text {de }}$ \\
\hline G2 & $35.23 \pm 1.86^{b c}$ & $34.30 \pm 0.93^{\mathrm{ab}}$ & $28.74 \pm 0.93^{a}$ & $26.88 \pm 0.93^{b}$ & $22.25 \pm 0.00^{\mathrm{bcd}}$ \\
\hline G3 & $32.45 \pm 3.71^{c}$ & $31.52 \pm 0.93^{b c}$ & $22.25 \pm 0.93^{\mathrm{bcd}}$ & $14.83 \pm 0.93^{d}$ & $11.12 \pm 0.00^{f}$ \\
\hline G4 & $32.45 \pm 2.78^{c}$ & $30.59 \pm 0.93^{\mathrm{bcd}}$ & $25.03 \pm 0.93^{a b c}$ & $20.40 \pm 0.00^{c}$ & $24.10 \pm 1.86^{\mathrm{ab}}$ \\
\hline G5 & $40.79 \pm 1.85^{b}$ & $28.74 \pm 0.93^{c d}$ & $29.67 \pm 0.00^{\mathrm{a}}$ & $26.88 \pm 0.93^{b}$ & $21.32 \pm 0.93^{\mathrm{bcd}}$ \\
\hline G6 & $39.86 \pm 0.93^{b}$ & $29.67 \pm 0.00^{c d}$ & $12.98 \pm 0.00^{e}$ & $18.54 \pm 0.00^{c}$ & $26.88 \pm 0.93^{a}$ \\
\hline G7 & $49.13 \pm 0.93^{a}$ & $27.81 \pm 1.86^{c d}$ & $30.59 \pm 0.93^{a}$ & $37.08 \pm 0.00^{\mathrm{a}}$ & $17.61 \pm 0.93^{e}$ \\
\hline G8 & $36.16 \pm 0.93^{b c}$ & $26.88 \pm 0.93^{d}$ & $18.54 \pm 1.86^{d}$ & $20.40 \pm 0.00^{c}$ & $20.40 \pm 0.00^{\text {cde }}$ \\
\hline C & $39.86 \pm 2.78^{b}$ & $31.52 \pm 0.00^{\mathrm{bc}}$ & $20.40 \pm 0.00^{c d}$ & $19.47 \pm 0.93^{c}$ & $23.18 \pm 0.93^{b c}$ \\
\hline
\end{tabular}

The values are mean values \pm SD $(n=3)$. Different lowercased letters in one column indicate a significant difference with $P<0.05$.

\section{Soil available $P$}

Table 7 presents that before wheat sowing, the treatment $\mathrm{S}$ held a higher trend of soil available $\mathrm{P}$ at $0-20 \mathrm{~cm}, 40-$ $60 \mathrm{~cm}, 60-80 \mathrm{~cm}$, and $80-100 \mathrm{~cm}$ than other treatments, which indicated that growing soy bean could also enhance the soil available $P$ at these soil layers. And the $0-20 \mathrm{~cm}$ soil available $\mathrm{P}$ of the treatment $\mathrm{S}$ was $18.09 \%-91.06 \%$ higher than other treatments. After wheat harvest, a higher trend of $0-20 \mathrm{~cm}$ soil available P belonged to G7 compared with other treatments. And the $0-20 \mathrm{~cm}$ available P of G7 was $2.06 \%-948.48 \%$ better than other treatments. G1 had the significantly highest available $\mathrm{P}$ of $20-40 \mathrm{~cm}$.

\section{Soil Total $P$}

Before wheat sowing, the treatment $\mathrm{C}$ held a lower trend of soil total P of five layers than other treatments (Table 8), implying that growing green manures could enhance $0-100 \mathrm{~cm}$ soil total $\mathrm{P}$ before wheat sowing. And the $0-20 \mathrm{~cm}$ soil total P of R1 was $10.99 \%-23.17 \%$ better than other treatments. After wheat harvest, G3 had the significantly highest soil total P of $0-20 \mathrm{~cm}$, which was $25 \%-45.83 \%$ better than other treatments. However, the treatment $\mathrm{C}$ had the lowest total P of $20-40 \mathrm{~cm}, 40-60 \mathrm{~cm}$, and $80-100 \mathrm{~cm}$, indicating that the application of green manures improved soil total $\mathrm{P}$ in these soil layers after wheat harvest.

\section{Soil available $K$}

Before wheat sowing, the treatment $C$ kept the lowest soil available $\mathrm{K}$ of five layers (Table 9), which indicated that growing green manures could improve $0-100 \mathrm{~cm}$ soil available $\mathrm{K}$. After wheat harvest, G8 led to a lower trend of soil 
Table 6: Soil total $\mathrm{N}(\mathrm{g} / \mathrm{kg})$ at two stages

\begin{tabular}{|c|c|c|c|c|c|}
\hline \multirow[t]{2}{*}{ Treatments } & \multicolumn{5}{|c|}{ Soil total $N(\mathrm{~g} / \mathrm{kg})$ before wheat sowing } \\
\hline & $0-20 \mathrm{~cm}$ & $20-40 \mathrm{~cm}$ & $40-60 \mathrm{~cm}$ & $60-80 \mathrm{~cm}$ & $80-100 \mathrm{~cm}$ \\
\hline $\mathrm{R} 1$ & $1.09 \pm 0.03^{a}$ & $0.97 \pm 0.04^{a}$ & $0.72 \pm 0.02^{\mathrm{a}}$ & $0.65 \pm 0.02^{c}$ & $0.70 \pm 0.05^{\mathrm{abc}}$ \\
\hline $\mathrm{R} 2$ & $0.93 \pm 0.03^{a}$ & $0.92 \pm 0.05^{\mathrm{a}}$ & $0.76 \pm 0.03^{a}$ & $0.75 \pm 0.03^{\mathrm{bc}}$ & $0.63 \pm 0.03^{\mathrm{bc}}$ \\
\hline R3 & $0.95 \pm 0.04^{a}$ & $0.89 \pm 0.01^{a}$ & $0.85 \pm 0.03^{\mathrm{a}}$ & $0.83 \pm 0.05^{\mathrm{ab}}$ & $0.73 \pm 0.01^{\mathrm{ab}}$ \\
\hline S & $1.05 \pm 0.01^{\mathrm{a}}$ & $0.95 \pm 0.07^{a}$ & $0.89 \pm 0.05^{a}$ & $0.94 \pm 0.03^{\mathrm{a}}$ & $0.75 \pm 0.04^{a}$ \\
\hline C & $0.94 \pm 0.01^{a}$ & $0.95 \pm 0.08^{a}$ & $0.87 \pm 0.06^{\mathrm{a}}$ & $0.73 \pm 0.04^{\mathrm{bc}}$ & $0.61 \pm 0.05^{c}$ \\
\hline \multicolumn{6}{|c|}{ Soil total $N(g / k g)$ after wheat harvest } \\
\hline Treatments & $0-20 \mathrm{~cm}$ & $20-40 \mathrm{~cm}$ & $40-60 \mathrm{~cm}$ & $60-80 \mathrm{~cm}$ & $80-100 \mathrm{~cm}$ \\
\hline G1 & $0.72 \pm 0.02^{b}$ & $0.62 \pm 0.02^{\mathrm{de}}$ & $0.52 \pm 0.00^{\mathrm{ab}}$ & $0.38 \pm 0.01^{d}$ & $0.40 \pm 0.01^{\text {cd }}$ \\
\hline G2 & $0.77 \pm 0.01^{b}$ & $0.69 \pm 0.01^{b c}$ & $0.53 \pm 0.01^{\mathrm{ab}}$ & $0.49 \pm 0.02^{b}$ & $0.40 \pm 0.01^{\mathrm{cd}}$ \\
\hline G3 & $0.68 \pm 0.16^{b}$ & $0.72 \pm 0.02^{b}$ & $0.55 \pm 0.01^{\mathrm{a}}$ & $0.53 \pm 0.01^{\mathrm{a}}$ & $0.50 \pm 0.01^{a}$ \\
\hline G4 & $0.71 \pm 0.01^{b}$ & $0.59 \pm 0.01^{e}$ & $0.50 \pm 0.02^{b c}$ & $0.49 \pm 0.02^{b}$ & $0.37 \pm 0.00^{d}$ \\
\hline G5 & $0.79 \pm 0.01^{b}$ & $0.77 \pm 0.01^{a}$ & $0.55 \pm 0.01^{\mathrm{a}}$ & $0.54 \pm 0.02^{\mathrm{a}}$ & $0.46 \pm 0.01^{\mathrm{ab}}$ \\
\hline G6 & $0.82 \pm 0.00^{b}$ & $0.58 \pm 0.00^{e f}$ & $0.50 \pm 0.00^{b c}$ & $0.41 \pm 0.00^{\text {cd }}$ & $0.43 \pm 0.02^{b c}$ \\
\hline G7 & $1.60 \pm 0.01^{a}$ & $0.66 \pm 0.02^{c d}$ & $0.50 \pm 0.00^{\text {bc }}$ & $0.27 \pm 0.00^{\mathrm{e}}$ & $0.47 \pm 0.02^{\mathrm{ab}}$ \\
\hline G8 & $0.75 \pm 0.01 b$ & $0.54 \pm 0.02^{f}$ & $0.46 \pm 0.01^{d}$ & $0.31 \pm 0.00^{e}$ & $0.30 \pm 0.01^{e}$ \\
\hline C & $0.75 \pm 0.01 b$ & $0.62 \pm 0.00^{\text {de }}$ & $0.48 \pm 0.01^{\mathrm{cd}}$ & $0.44 \pm 0.01^{c}$ & $0.50 \pm 0.01^{\mathrm{a}}$ \\
\hline
\end{tabular}

The values are mean values \pm SD $(n=3)$. Different lowercased letters in one column indicate a significant difference with $P<0.05$.

Table 7: Soil available $\mathbf{P}(\mathrm{mg} / \mathrm{kg})$ at two stages

\begin{tabular}{|c|c|c|c|c|c|}
\hline \multicolumn{6}{|c|}{ Soil available $P(\mathrm{mg} / \mathrm{kg})$ before wheat sowing } \\
\hline Treatments & $0-20 \mathrm{~cm}$ & $20-40 \mathrm{~cm}$ & $40-60 \mathrm{~cm}$ & $60-80 \mathrm{~cm}$ & $80-100 \mathrm{~cm}$ \\
\hline R1 & $1.99 \pm 0.04^{\mathrm{ab}}$ & $1.54 \pm 0.04^{a}$ & $0.68 \pm 0.01^{b}$ & $0.87 \pm 0.01^{a}$ & $0.65 \pm 0.04^{a}$ \\
\hline $\mathrm{R} 2$ & $1.23 \pm 0.19^{c}$ & $0.29 \pm 0.00^{c}$ & $0.26 \pm 0.01^{c}$ & $1.20 \pm 0.01^{\mathrm{a}}$ & $1.28 \pm 0.04^{\mathrm{a}}$ \\
\hline R3 & $1.66 \pm 0.03^{\mathrm{bc}}$ & $1.20 \pm 0.04^{\mathrm{ab}}$ & $1.01 \pm 0.06^{\mathrm{a}}$ & $0.77 \pm 0.06^{a}$ & $0.86 \pm 0.06^{a}$ \\
\hline S & $2.35 \pm 0.00^{\mathrm{a}}$ & $1.28 \pm 0.03^{\mathrm{ab}}$ & $1.23 \pm 0.02^{\mathrm{a}}$ & $1.05 \pm 0.02^{\mathrm{a}}$ & $1.62 \pm 0.00^{\mathrm{a}}$ \\
\hline C & $1.81 \pm 0.04^{\mathrm{abc}}$ & $0.60 \pm 0.02^{\mathrm{bc}}$ & $0.48 \pm 0.03^{\mathrm{bc}}$ & $0.31 \pm 0.03^{a}$ & $0.28 \pm 0.06^{a}$ \\
\hline \multicolumn{6}{|c|}{ Soil available $P(\mathrm{mg} / \mathrm{kg})$ after wheat harvest } \\
\hline Treatments & $0-20 \mathrm{~cm}$ & $20-40 \mathrm{~cm}$ & $40-60 \mathrm{~cm}$ & $60-80 \mathrm{~cm}$ & $80-100 \mathrm{~cm}$ \\
\hline G1 & $3.39 \pm 0.06^{\mathrm{ab}}$ & $1.79 \pm 0.03^{a}$ & $0.84 \pm 0.03^{a}$ & $0.77 \pm 0.04^{\mathrm{ab}}$ & $0.76 \pm 0.06^{a}$ \\
\hline G2 & $0.33 \pm 0.02^{c}$ & $0.31 \pm 0.02^{c}$ & $0.29 \pm 0.03^{b}$ & $0.26 \pm 0.03^{c}$ & $0.33 \pm 0.10^{\mathrm{abc}}$ \\
\hline G3 & $3.00 \pm 0.03^{\mathrm{abc}}$ & $0.89 \pm 0.01^{\mathrm{bc}}$ & $0.66 \pm 0.05^{a}$ & $0.32 \pm 0.02^{\mathrm{ab}}$ & $0.36 \pm 0.00^{\mathrm{abc}}$ \\
\hline G4 & $1.00 \pm 0.02^{\mathrm{bc}}$ & $0.61 \pm 0.04^{\mathrm{bc}}$ & $0.40 \pm 0.03^{b}$ & $0.30 \pm 0.02^{c}$ & $0.31 \pm 0.00^{b c}$ \\
\hline G5 & $0.94 \pm 0.02^{\mathrm{bc}}$ & $1.00 \pm 0.04^{b}$ & $0.51 \pm 0.01^{b}$ & $0.81 \pm 0.06^{b c}$ & $0.72 \pm 0.05^{\mathrm{ab}}$ \\
\hline G6 & $0.72 \pm 0.04^{c}$ & $0.34 \pm 0.04^{c}$ & $0.45 \pm 0.05^{b}$ & $0.30 \pm 0.02^{c}$ & $0.22 \pm 0.04^{c}$ \\
\hline G7 & $3.46 \pm 0.02^{\mathrm{a}}$ & $0.63 \pm 0.04^{\mathrm{bc}}$ & $0.46 \pm 0.01^{b}$ & $0.29 \pm 0.04^{c}$ & $0.34 \pm 0.02^{\mathrm{abc}}$ \\
\hline G8 & $0.95 \pm 0.04^{b c}$ & $0.76 \pm 0.02^{\mathrm{bc}}$ & $0.53 \pm 0.03^{b}$ & $0.43 \pm 0.00^{c}$ & $0.50 \pm 0.03^{\mathrm{abc}}$ \\
\hline C & $3.24 \pm 0.06^{\mathrm{ab}}$ & $1.00 \pm 0.03^{b}$ & $0.50 \pm 0.04^{b}$ & $0.88 \pm 0.00^{\mathrm{a}}$ & $0.62 \pm 0.05^{\mathrm{abc}}$ \\
\hline
\end{tabular}

The values are mean values \pm SD $(n=3)$. Different lowercased letters in one column indicate a significant difference with $P<0.05$.

available $\mathrm{K}$ of five layers compared with other treatments. At $0-20 \mathrm{~cm}$, the soil available K of G8 was $21.81 \%-125.87 \%$ lower than that of other treatments. In this study, the available $\mathrm{K}$ of five layers of all the treatments was decreased after wheat harvest compared to them before wheat sowing (data not shown). And the reduction of soil available $\mathrm{K}$ of five layers under the green manures treatments was much more than that under the treatment $C$ (data not shown).

\section{Soil organic matter}

Before wheat sowing, the significantly highest soil organic matter of $0-20 \mathrm{~cm}$ and $20-40 \mathrm{~cm}$ belonged to the treatment $\mathrm{S}$ compared with other treatments (Table 10). At 0-20 cm, the soil organic matter of the treatment $\mathrm{S}$ was $40.28 \%$ $71.12 \%$ higher than other treatments. And at $20-40 \mathrm{~cm}$, the soil organic matter of the treatment $\mathrm{S}$ was $53.92 \%$ $122.67 \%$ better compared with other treatments. After wheat harvest, there was no significant difference between the soil organic matter of green manures treatments and that of the treatment $C$ from $0 \mathrm{~cm}$ to $100 \mathrm{~cm}$.

\section{DISCUSSION}

\section{Grain yield of winter wheat}

In this study, the treatment $C$ held the highest grain yield than the green manures treatments. Therefore, the rotation and incorporation of green manures during the fallow period decreased subsequent wheat yield (Table 3), which was contrary with a previous study (Hossain et al., 2016). 
Table 8: Soil total $P(\mathrm{~g} / \mathrm{kg})$ at two stages

\begin{tabular}{|c|c|c|c|c|c|}
\hline \multicolumn{6}{|c|}{ Soil total $P(\mathrm{~g} / \mathrm{kg})$ before wheat sowing } \\
\hline Treatments & $0-20 \mathrm{~cm}$ & $20-40 \mathrm{~cm}$ & $40-60 \mathrm{~cm}$ & $60-80 \mathrm{~cm}$ & $80-100 \mathrm{~cm}$ \\
\hline $\mathrm{R} 1$ & $1.01 \pm 0.08^{a}$ & $0.95 \pm 0.01^{a}$ & $0.81 \pm 0.04^{a}$ & $0.80 \pm 0.05^{\mathrm{a}}$ & $0.82 \pm 0.04^{a}$ \\
\hline $\mathrm{R} 2$ & $0.91 \pm 0.05^{\mathrm{ab}}$ & $0.91 \pm 0.02^{\mathrm{ab}}$ & $0.79 \pm 0.01^{\mathrm{ab}}$ & $0.76 \pm 0.03^{\mathrm{ab}}$ & $0.72 \pm 0.02 b^{c}$ \\
\hline R3 & $0.88 \pm 0.03^{\mathrm{ab}}$ & $0.86 \pm 0.04^{\mathrm{ab}}$ & $0.80 \pm 0.01^{\mathrm{a}}$ & $0.74 \pm 0.05^{\mathrm{ab}}$ & $0.74 \pm 0.02^{b}$ \\
\hline S & $0.85 \pm 0.01^{\mathrm{b}}$ & $0.78 \pm 0.06^{\mathrm{bc}}$ & $0.73 \pm 0.02^{b}$ & $0.69 \pm 0.06^{b c}$ & $0.68 \pm 0.03^{\mathrm{bc}}$ \\
\hline C & $0.82 \pm 0.02^{\mathrm{b}}$ & $0.64 \pm 0.07^{c}$ & $0.63 \pm 0.05^{c}$ & $0.59 \pm 0.05^{c}$ & $0.65 \pm 0.03^{c}$ \\
\hline \multicolumn{6}{|c|}{ Soil total $P(g / k g)$ after wheat harvest } \\
\hline Treatments & $0-20 \mathrm{~cm}$ & $20-40 \mathrm{~cm}$ & $40-60 \mathrm{~cm}$ & $60-80 \mathrm{~cm}$ & $80-100 \mathrm{~cm}$ \\
\hline G1 & $0.84 \pm 0.02^{b}$ & $0.77 \pm 0.03^{a}$ & $0.71 \pm 0.00^{\mathrm{a}}$ & $0.73 \pm 0.02^{\mathrm{a}}$ & $0.78 \pm 0.01^{a}$ \\
\hline G2 & $0.78 \pm 0.01^{b}$ & $0.74 \pm 0.01^{\mathrm{a}}$ & $0.69 \pm 0.01^{a b}$ & $0.70 \pm 0.01^{\mathrm{ab}}$ & $0.64 \pm 0.01^{\mathrm{cd}}$ \\
\hline G3 & $1.05 \pm 0.01^{a}$ & $0.75 \pm 0.01^{a}$ & $0.71 \pm 0.01^{a}$ & $0.67 \pm 0.00^{b c}$ & $0.69 \pm 0.01^{b}$ \\
\hline G4 & $0.74 \pm 0.00^{b}$ & $0.73 \pm 0.03^{\mathrm{ab}}$ & $0.67 \pm 0.01^{b c}$ & $0.64 \pm 0.01^{\mathrm{cd}}$ & $0.66 \pm 0.01^{\mathrm{bcd}}$ \\
\hline G5 & $0.84 \pm 0.00^{b}$ & $0.78 \pm 0.00^{a}$ & $0.71 \pm 0.00^{a}$ & $0.71 \pm 0.01^{\mathrm{ab}}$ & $0.67 \pm 0.01^{\mathrm{bc}}$ \\
\hline G6 & $0.75 \pm 0.00^{b}$ & $0.65 \pm 0.00^{c}$ & $0.64 \pm 0.01^{d}$ & $0.60 \pm 0.02^{d}$ & $0.63 \pm 0.01^{d}$ \\
\hline G7 & $0.84 \pm 0.01^{b}$ & $0.69 \pm 0.01 b^{c}$ & $0.63 \pm 0.00^{d}$ & $0.62 \pm 0.01^{d}$ & $0.59 \pm 0.01^{\mathrm{e}}$ \\
\hline G8 & $0.72 \pm 0.00^{b}$ & $0.66 \pm 0.01^{c}$ & $0.65 \pm 0.01 c^{d}$ & $0.67 \pm 0.01^{b c}$ & $0.64 \pm 0.01^{\mathrm{cd}}$ \\
\hline C & $0.82 \pm 0.02^{b}$ & $0.50 \pm 0.00^{d}$ & $0.56 \pm 0.02^{\mathrm{e}}$ & $0.60 \pm 0.02^{\mathrm{d}}$ & $0.54 \pm 0.01^{f}$ \\
\hline
\end{tabular}

The values are mean values \pm SD $(n=3)$. Different lowercased letters in one column indicate a significant difference with $P<0.05$.

Table 9: Soil available $\mathrm{K}(\mathrm{mg} / \mathrm{kg})$ at two stages

\begin{tabular}{|c|c|c|c|c|c|}
\hline \multicolumn{6}{|c|}{ Soil available $\mathrm{K}(\mathrm{mg} / \mathrm{kg})$ before wheat sowing } \\
\hline Treatments & $0-20 \mathrm{~cm}$ & $20-40 \mathrm{~cm}$ & $40-60 \mathrm{~cm}$ & $60-80 \mathrm{~cm}$ & $80-100 \mathrm{~cm}$ \\
\hline $\mathrm{R} 1$ & $858.39 \pm 16.70^{\mathrm{a}}$ & $836.31 \pm 31.40^{\mathrm{a}}$ & $809.70 \pm 28.75^{\mathrm{ab}}$ & $766.24 \pm 29.77^{b}$ & $772.97 \pm 35.69^{b}$ \\
\hline $\mathrm{R} 2$ & $890.18 \pm 10.19^{a}$ & $822.24 \pm 11.77^{a}$ & $720.33 \pm 32.76^{b}$ & $689.75 \pm 11.77^{b}$ & $679.56 \pm 11.77^{b}$ \\
\hline R3 & $907.16 \pm 29.42^{\mathrm{a}}$ & $903.76 \pm 25.65^{a}$ & $869.79 \pm 17.65^{a}$ & $890.18 \pm 26.96^{\mathrm{a}}$ & $886.78 \pm 11.77^{\mathrm{a}}$ \\
\hline S & $847.71 \pm 22.33^{a}$ & $832.43 \pm 10.05^{a}$ & $764.49 \pm 10.92^{\mathrm{ab}}$ & $788.27 \pm 13.43^{\mathrm{ab}}$ & $754.30 \pm 10.02^{b}$ \\
\hline C & $540.28 \pm 45.95^{b}$ & $475.74 \pm 21.21^{b}$ & $468.95 \pm 5.88^{c}$ & $462.15 \pm 10.19^{c}$ & $445.17 \pm 32.76^{c}$ \\
\hline \multicolumn{6}{|c|}{ Soil available $\mathrm{K}(\mathrm{mg} / \mathrm{kg})$ after wheat harvest } \\
\hline Treatments & $0-20 \mathrm{~cm}$ & $20-40 \mathrm{~cm}$ & $40-60 \mathrm{~cm}$ & $60-80 \mathrm{~cm}$ & $80-100 \mathrm{~cm}$ \\
\hline G1 & $174.99 \pm 10.08^{a b}$ & $144.88 \pm 0.17^{\mathrm{ab}}$ & $111.55 \pm 7.06^{\mathrm{abc}}$ & $100.99 \pm 3.61^{\mathrm{ab}}$ & $95.08 \pm 3.09^{b c}$ \\
\hline G2 & $320.29 \pm 8.25^{a}$ & $141.61 \pm 3.66^{\mathrm{ab}}$ & $137.70 \pm 5.86^{a}$ & $111.41 \pm 0.93^{a}$ & $108.90 \pm 2.47^{\mathrm{ab}}$ \\
\hline G3 & $201.90 \pm 2.60^{\mathrm{ab}}$ & $131.35 \pm 10.76^{\mathrm{ab}}$ & $107.73 \pm 16.87^{b c}$ & $84.43 \pm 0.77^{b}$ & $89.59 \pm 2.09^{b c}$ \\
\hline G4 & $175.16 \pm 4.70^{\mathrm{ab}}$ & $148.94 \pm 9.93^{a}$ & $121.85 \pm 5.07^{\mathrm{abc}}$ & $100.09 \pm 8.79^{a b}$ & $98.20 \pm 7.26 \mathrm{a}^{\mathrm{bc}}$ \\
\hline G5 & $211.99 \pm 6.22^{\mathrm{ab}}$ & $141.27 \pm 1.54^{\mathrm{ab}}$ & $126.90 \pm 14.46^{\mathrm{ab}}$ & $112.39 \pm 0.09^{a}$ & $115.49 \pm 0.05^{a}$ \\
\hline G6 & $172.73 \pm 2.76^{\mathrm{ab}}$ & $136.28 \pm 0.69^{a b}$ & $114.43 \pm 1.61^{\mathrm{abc}}$ & $108.79 \pm 8.81^{a}$ & $108.29 \pm 12.13^{a b}$ \\
\hline G7 & $177.57 \pm 1.75^{\mathrm{ab}}$ & $141.19 \pm 3.02^{\mathrm{ab}}$ & $114.59 \pm 4.59^{b c}$ & $91.61 \pm 7.85^{\mathrm{ab}}$ & $83.34 \pm 8.77^{c}$ \\
\hline G8 & $141.80 \pm 1.99^{b}$ & $105.13 \pm 6.08^{c}$ & $94.54 \pm 0.52^{c}$ & $83.29 \pm 4.54 b$ & $82.74 \pm 0.24^{c}$ \\
\hline C & $176.46 \pm 7.66^{\mathrm{ab}}$ & $126.97 \pm 5.64^{b}$ & $111.22 \pm 2.28^{\mathrm{abc}}$ & $109.12 \pm 10.46^{a}$ & $94.10 \pm 0.08^{b c}$ \\
\hline
\end{tabular}

The values are mean values $\pm \mathrm{SD}(\mathrm{n}=3)$. Different lowercased letters in one column indicate a significant difference with $P<0.05$.

This may be resulted from the extremely limited precipitation during the summer fallow from 2015 to 2016, which was only $94.7 \mathrm{~mm}$ (Fig. 1). In addition, the treatment $\mathrm{C}$ led to a higher trend of soil relative water content of $0-100 \mathrm{~cm}$ than the green manures treatments before wheat sowing (Table 4). Sufficient soil water supply promotes seed germination, tiller growth, and spike differentiation of the winter wheat (Musick et al., 1994; Zhou et al., 2009, 2011). This might explain the highest grain yield was found in the treatment $\mathrm{C}$ (Table 3 ).

\section{Soil relative water content}

Regarding to soil water content, a higher trend of $0-100 \mathrm{~cm}$ water content belonged to the treatment $\mathrm{C}$ compared with the green manures treatments (Table 4). Furthermore, the $0-100 \mathrm{~cm}$ soil water content of the treatment $S$ showed a higher trend than that of R3. Due to the limited precipitation $(94.7 \mathrm{~mm})$ of fallow period from 2015 to 2016 (Fig. 1), planting green manures consumed much more soil water than bare field. Rapeseed had larger above ground biomass than soy bean (data not shown). And the rapeseed above ground biomass increased as the sowing rate was enhanced (data not shown). Therefore, planting rapeseed exploited more soil water than growing soy bean, especially the highest sowing rate of rapeseed.

\section{Soil nutrients}

\section{Soil available $N$ and total $N$}

The treatment $\mathrm{S}$ obtained a higher trend of available $\mathrm{N}$ of $0-60 \mathrm{~cm}$ than other treatments before wheat sowing 
Table 10: Soil organic matter (\%) at two stages

\begin{tabular}{|c|c|c|c|c|c|}
\hline \multicolumn{6}{|c|}{ Soil organic matter (\%) before wheat sowing } \\
\hline Treatments & $0-20 \mathrm{~cm}$ & $20-40 \mathrm{~cm}$ & $40-60 \mathrm{~cm}$ & $60-80 \mathrm{~cm}$ & $80-100 \mathrm{~cm}$ \\
\hline $\mathrm{R} 1$ & $2.37 \pm 0.11^{\mathrm{b}}$ & $1.89 \pm 0.11^{\mathrm{bc}}$ & $0.60 \pm 0.14^{b}$ & $0.86 \pm 0.36^{b}$ & $1.30 \pm 0.19^{a}$ \\
\hline $\mathrm{R} 2$ & $2.32 \pm 0.08^{b}$ & $1.50 \pm 0.00^{c}$ & $0.76 \pm 0.08^{b}$ & $0.49 \pm 0.07^{b}$ & $0.37 \pm 0.10^{b}$ \\
\hline R3 & $2.37 \pm 0.08^{b}$ & $2.17 \pm 0.09^{b}$ & $2.03 \pm 0.17^{\mathrm{a}}$ & $1.34 \pm 0.01^{\mathrm{ab}}$ & $1.42 \pm 0.07^{\mathrm{a}}$ \\
\hline S & $3.97 \pm 0.08^{\mathrm{a}}$ & $3.34 \pm 0.15^{\mathrm{a}}$ & $2.66 \pm 0.18^{\mathrm{a}}$ & $2.03 \pm 0.10^{\mathrm{a}}$ & $1.65 \pm 0.17^{\mathrm{a}}$ \\
\hline C & $2.83 \pm 0.20^{\mathrm{b}}$ & $2.12 \pm 0.16^{b}$ & $2.25 \pm 0.19^{\mathrm{a}}$ & $0.95 \pm 0.05^{b}$ & $1.48 \pm 0.18^{\mathrm{a}}$ \\
\hline \multicolumn{6}{|c|}{ Soil organic matter $(\%)$ during wheat harvest } \\
\hline Treatments & $0-20 \mathrm{~cm}$ & $20-40 \mathrm{~cm}$ & $40-60 \mathrm{~cm}$ & $60-80 \mathrm{~cm}$ & $80-100 \mathrm{~cm}$ \\
\hline G1 & $2.58 \pm 0.16^{\mathrm{ab}}$ & $1.61 \pm 0.14^{b}$ & $1.56 \pm 0.23^{\mathrm{abc}}$ & $1.08 \pm 0.27^{a}$ & $1.28 \pm 0.05^{\mathrm{ab}}$ \\
\hline G2 & $2.48 \pm 0.07^{\mathrm{ab}}$ & $2.03 \pm 0.24^{\mathrm{ab}}$ & $1.68 \pm 0.25^{\mathrm{ab}}$ & $1.37 \pm 0.22^{\mathrm{a}}$ & $1.36 \pm 0.28^{\mathrm{ab}}$ \\
\hline G3 & $3.25 \pm 0.14^{\mathrm{a}}$ & $2.60 \pm 0.29^{a}$ & $1.98 \pm 0.19^{a}$ & $1.35 \pm 0.24^{a}$ & $1.68 \pm 0.15^{a}$ \\
\hline G4 & $3.06 \pm 0.15^{\mathrm{ab}}$ & $2.47 \pm 0.10^{\mathrm{ab}}$ & $1.29 \pm 0.10 a^{b c}$ & $1.34 \pm 0.27^{a}$ & $1.20 \pm 0.18^{\mathrm{abc}}$ \\
\hline G5 & $3.00 \pm 0.17^{\mathrm{ab}}$ & $2.28 \pm 0.16^{\mathrm{ab}}$ & $1.67 \pm 0.35^{\mathrm{ab}}$ & $0.90 \pm 0.21^{a}$ & $0.94 \pm 0.20^{\mathrm{bc}}$ \\
\hline G6 & $2.08 \pm 0.15^{b}$ & $1.66 \pm 0.25^{b}$ & $1.17 \pm 0.43^{\mathrm{bc}}$ & $1.20 \pm 0.15^{\mathrm{a}}$ & $1.00 \pm 0.20^{\mathrm{bc}}$ \\
\hline G7 & $2.63 \pm 0.15^{\mathrm{ab}}$ & $2.21 \pm 0.17^{\mathrm{ab}}$ & $1.23 \pm 0.16 a^{b c}$ & $1.07 \pm 0.17^{\mathrm{a}}$ & $1.04 \pm 0.29^{\mathrm{bc}}$ \\
\hline G8 & $2.40 \pm 0.13^{\mathrm{ab}}$ & $1.74 \pm 0.30^{\mathrm{ab}}$ & $0.86 \pm 0.12^{c}$ & $1.18 \pm 0.12^{\mathrm{a}}$ & $0.83 \pm 0.20^{\mathrm{bc}}$ \\
\hline $\mathrm{C}$ & $2.63 \pm 0.13^{\mathrm{ab}}$ & $1.61 \pm 0.13^{b}$ & $1.30 \pm 0.16 a b^{c}$ & $1.03 \pm 0.30^{\mathrm{a}}$ & $0.63 \pm 0.10^{c}$ \\
\hline
\end{tabular}

The values are mean values \pm SD $(n=3)$. Different lowercased letters in one column indicate a significant difference with $P<0.05$.

(Table 5). After wheat harvest, G7 led to the highest soil available $\mathrm{N}$ of $0-20 \mathrm{~cm}$ (Table 5). Before wheat sowing, the treatment $\mathrm{S}$ held a higher trend of soil total $\mathrm{N}$ from 20 to $100 \mathrm{~cm}$ compared with other treatments (Table 6). And G7 led to the highest soil total $\mathrm{N}$ of $0-20 \mathrm{~cm}$ after wheat harvest (Table 6). All these results were consistent with a previous study (Xie et al., 2016). This may be resulted from that legumes could promote soil physical and biological properties (e.g. urease activity and microbial biomass) and enhance soil N cycling (Tejada et al., 2008; Lee et al., 2010; Piotrowska and Wilczewski, 2012). In addition, legumes could also improve soil $\mathrm{N}$ supply for subsequent crops by the return and mineralization of $\mathrm{N}$-rich above-and below-ground crop residues (Beckie and Brandt, 1997; Arcand et al., 2014). Therefore, the treatments $\mathrm{S}$ and $\mathrm{G} 7$ could enhance soil available $\mathrm{N}$ and total N.

\section{Soil available $P$ and total $P$}

The treatment $S$ led to a higher trend of soil available $P$ at $0-20 \mathrm{~cm}$ and $40-100 \mathrm{~cm}$ than other treatments before wheat sowing (Table 7). After wheat harvest, a higher trend of soil available $P$ at $0-20 \mathrm{~cm}$ belonged to $\mathrm{G} 7$ compared with other treatment (Table 7). A lower trend of soil total $\mathrm{P}$ of five layers belonged to the treatment $\mathrm{C}$ than other treatments before wheat sowing (Table 8). After wheat harvest, G3 had the significantly highest soil total P of $0-20 \mathrm{~cm}$ (Table 8). Therefore, the insertion of green manures could also increase the $\mathrm{P}$ content in the soil, which is supported by a previous study (Vanlauwe et al., 2000). During the green manures' growing period, they could mobilize relatively unavailable $\mathrm{P}$ forms owing to the excretion of P-solublizing compounds in the rhizosphere (Schilling et al., 1998). After decomposition, the $\mathrm{P}$ is released and the green manures could improve the P nutrition status of the soil (EichlerLöbermann et al., 2008).

\section{Soil available $K$}

Before wheat sowing, all the green manures treatments kept significantly higher soil available $\mathrm{K}$ of five layers than that of the treatment $C$ (Table 9). Maybe the soil erosion from rainfall during summer fallow resulted in the low available $\mathrm{K}$ in the treatment $\mathrm{C}$. Due to the continental monsoon climate of this region, more than $60 \%$ of the annual precipitation falls during the wheat fallow period (Jin et al., 2007). In this study, the available K of five layers of all the treatments was decreased after wheat harvest compared to them before wheat sowing (data not shown). And the reduction of soil available $\mathrm{K}$ of five layers under the green manures treatments was much more than that under the treatment $\mathrm{C}$ (data not shown). This may be mainly owing to heavy removal of $\mathrm{K}$ by all the treatments, especially the green manures treatments. The removal of $\mathrm{K}$ was greater than its application in this area and the contribution from non-exchangeable to exchangeable $\mathrm{K}$ in soil was insufficient. Moreover, the application of $\mathrm{K}$ to wheat and green manures could not meet the demand for $\mathrm{K}$. This hypothesis is supported by some precious research (Singh et al., 2004; Panaullah et al., 2006).

\section{Soil organic matter}

The treatment $\mathrm{S}$ only obtained the significantly highest soil organic matter of $0-20 \mathrm{~cm}$ and $20-40 \mathrm{~cm}$ before wheat sowing (Table 10). However, after wheat harvest, all the green manures treatments obtained no significantly better soil organic matter of five layers than that of the treatment C (Table 10). Thus the incorporation of green manures had not enhanced the soil organic matter after wheat harvest. 
Some longer experiments also had the same results. The impact of green manures treatments on organic carbon had no influence during the three-year-long experimental time (Piotrowska and Wilczewski, 2012). Eichler-Löbermann et al. (2008) found that different green manures did not significantly improve the organic matter content in a period of three years. In addition, the high organic matter input of pig slurry and green manures of 8 years had no effect on soil total carbon (Debosz et al., 1999). The reason may be that the amount of organic matter accumulation in soil can vary depending mainly on decomposition rate and the type of green manures (Egodawatta et al., 2012). Also, green manure decomposition and subsequent nutrients release depend largely on soil physical (moisture, temperature, texture, mineralogy and acidity), chemical $(\mathrm{C} / \mathrm{N}$ ratio, presence of nutrients) and biological activity (Myers et al., 1994).

Due to the limited experimental time (one year) and relatively dry weather condition, growing green manures during fallow period in this region decreased wheat grain yield and not all the green manures treatments improved soil nutrients of $0-100 \mathrm{~cm}$. Therefore, a long period experiment should be carried out in the future.

\section{CONCLUSIONS}

Growing rapeseed and soy bean during summer fallow in this region significantly reduced 1,000-grain weight and grain yield of subsequent winter wheat relative to the bare field. But the incorporation of green manures improved the soil nutrients to some extent.

\section{ACKNOWLEDGMETS}

This study was financially supported by the Special Fund for Agro-scientific Research in the Public Interest (201503120), the National Natural Science Foundation of China (31101113), the Special Fund for the Construction of Modern Agricultural Industry Technology System (CARS-03-01-24), the Fund for Shanxi 1331 Project, Loess Plateau crops with high quality and efficient production Shanxi Province Collaborative Innovation Center (Shanxi Educational Fund [2016]75).

\section{Author contributions}

Zhenping Yang designed the study. Zhiqiang Gao provided the financial support for the study. Chunlai Zhang provided the lab for the experiments. Xiaoli Liu and Naiwen Xue collected samples and finished the experiments. Naiwen Xue drafted the manuscript. Min Sun and Tianqing Du helped analyze the experimental data. Jianfu Xue helped review the structure and language of this manuscript.

\section{REFERENCES}

Arcand, M. M., R. Lemke, R. E. Farrell and J. D. Knight. 2014. Nitrogen supply from below-ground residues of lentil and wheat to a subsequent wheat crop. Biol. Fertil. Soils. 50: 507-515.

Beckie, H. J. and S. A. Brandt. 1997. Nitrogen contribution of field pea in annual cropping systems 1 . Nitrogen residual effect. Can. J. Plant Sci. 77: 311-322.

Debosz, K., P. H. Rasmussen and A. R. Pedersen. 1999. Temporal variations in microbial Biomass $\mathrm{C}$ and cellulolytic enzyme activity in arable soils: Effects of organic matter input. Appl. Soil Ecol. 13: 209-218.

Egodawatta, W. C. P., U. R. Sangakkara and P. Stamp. 2012. Impact of green manure and mineral fertilizer inputs on soil organic matter and crop productivity in a sloping landscape of Sri Lanka. Field Crops Res. 129: 21-27.

Eichler-Löbermann, B., S. Köhne, B. Kowalski and E. Schnug. 2008. Effect of catch cropping on phosphorus bioavailability in comparison to organic and inorganic fertilization. J. Plant Nutr. 31: 659-676.

Hargrove, W. L. 1986. Winter legumes as a nitrogen source for no-till grain sorghum. Agron. J. 78: 70-74.

Hossain, M. S., A. Hossain, M. A. R. Sarkar, M. Jahiruddin, J. A. da Silva and T. M. I. Hossain. 2016. Productivity and soil fertility of the rice-wheat system in the high Ganges river floodplain of Bangladesh is influenced by the inclusion of legumes and manure. Agric. Ecosyst. Environ. 218: 40-52.

Hu, T., H. Li, Q. Cao, C. Yu, W. Cao and Y. Gao. 2015. Effects of green manure variety and growth density on forage value and soil fertility in dry land. J. Northwest AF Univ. 43(4): 44-52.

Hwang, H. Y., G. W. Kim, Y. B. Lee, P. J. Kim and S. Y. Kim. 2015. Improvement of the value of green manure via mixed hairy vetch and barley cultivation in temperate paddy soil. Field Crops Res. 183: $138-146$.

Jin, K., W. M. Cornelis, W. Schiettecatte, J. J. Lu, Y. Q. Yao, H. J. Wu, D. Gabriels, S. D. Neve, D. X. Cai, J. Y. Jin and R. Hartmann. 2007. Effects of different management practices on the soil water balance and crop yield for improved dryland farming in the Chinese Loess Plateau. Soil Tillage Res. 96: 131-144.

Krishnan, G., D. L. Holshouser and S. J. Nissen. 1998. Weed control in soybean (Glycinemax) with green manure crops. Weed Technol. 12: 97-102.

Lee, C. H., K. D. Park, K. Y. Jung, A. A. Muhammad, D. Lee, G. Jessie and J. K. Pil. 2010. Effect of Chinese milk vetch (Astragalus sinicus L.) as a green manure on rice productivity and methane emission in paddy soil. Agric. Ecosyst. Environ. 138: 343-347.

Li, S. X., Z. H. Wang, T. T. Hu, Y. J. Gao and B. A. Stewart. 2009. Nitrogen in dryland soils of China and its management. Adv. Agron. 101: 123-181.

Lu, R. K. 2000. Soil Agro-Chemical Analyses. Agricultural Technical Press of China, Beijing, China, p. 50.

Myers, R. J. K., C. A. Palm, E. Cuevas and I. U. N. Gunatilleke. 1994 The synchronization of nutrient mineralization and plant nutrient demand. In: Woomer, P. L., M. J. Swift (Eds.), The Biological Management of Tropical Soil Fertility. Wiley-Sayce Publication, Chichester, UK, pp. 81-116.

Norsworthy, J. K., L. Brandenberger, N. R. Burgos and M. Riley. 2005. Weeds uppression in Vigna unguiculata with a spring-seeded brassicaceae greenmanure. Crop Prot. 5: 441-447.

Panaullah, G. M., J. Timsina, M. A. Saleque, M. Ishaque, A. B. M. B. U. Pathan, D. J. Connor, P. K. Saha, M. A. Quayyum, E. Humphreys and C. A. Meisner. 2006. Nutrient uptake and 
apparent balances for rice-wheat sequences. III. Potassium. J. Plant Nutr. 29: 173-187.

Piotrowska, A. and E. Wilczewski. 2012. Effects of catch crops cultivated for green manure and mineral nitrogen fertilization on soil enzyme activities and chemical properties. Geoderma. 189190: $72-80$.

Poeplau, C., H. Aronsson, A. Myrbeck, T. Katterer. 2015. Effect of perennial ryegrass cover crop on soil organic carbon stocks in southern Sweden. Geoderma Reg. 4: 126-133.

Schilling, G., A. Gransee, A. Deubel, G. Lezovic and S. Ruppel. 1998. Phosphorus availability, root exudates, and microbial activity in the rhizosphere. Z. Pflanzenernährung Bodenkdunde. 161: 465-478.

Singh, Y., B. Singh, J. K. Ladha, C. S. Khind, R. K. Gupta, O. P. Meelu and E. Pasuquin. 2004. Long-term effects of organic inputs on yield and soil fertility in the rice-wheat rotation. Soil Sci. Soc. Am. J. 68: 845-853.

Snyder, D. and J. A. Trofymow. 1984. Rapid accurate wet oxidation diffusion procedure for determining organic and inorganic carbon in plant and soil samples. Commun. Soil Sci. Plant Anal. 15: $1587-1597$
Tang, K. L. (Ed.). 2004. Soil Erosion in China. Science and Technology Press, Beijing, China, p. 845.

Tejada, M., J. L. Gonzalez, A. M. García-Martínez and J. Parrado. 2008. Effects of different green manures on soil biological properties and maize yield. Bioresour. Technol. 99: 1758-1767.

Thorup-Kristensen, K., J. Magid and L. S. Jensen. 2003. Catch crops and green manures as biological tools in nitrogen management in temperate zones. Adv. Agronomy. 79: 227-302.

Vanlauwe, B., O. C. Nwoke, J. Diels, N. Sanginga, R. J. Carsky, J. Deckers, R. Merckx. 2000. Utilization of rock phosphate by crops and a representative toposequence in the Nothern Guinea savanna zone of Nigeria: Response by Mucuna pruriens, Lablab purpureus, and maize. Soil Biol. Biochem. 32: 2063-2077.

Vos, J. and P. van der Putten. 2001. Field observation on nitrogen catch crops. III. Transfer of nitrogen to the succeeding main crop. Plant Soil. 236: 263-273.

Xie, Z., S. Tu, F. Shah, C. Xu, J. Chen, D. Han, G. Liu, H. Li, I. Muhammad and W. Cao. 2016. Substitution of fertilizer-N by green manure improves the sustainability of yield in doublerice cropping system in south China. Field Crops Res. 188: $142-149$. 